\title{
Semipolar (10-11) GaN growth on silicon-on- insulator substrates: Defect reduction and meltback etching suppression
}

Cite as: J. Appl. Phys. 125, 035703 (2019); https://doi.org/10.1063/1.5067375

Submitted: 17 October 2018 . Accepted: 01 January 2019 . Published Online: 16 January 2019

Rami Mantach (D), P. Vennéguès, J. Zuniga Perez (D), P. De Mierry, M. Leroux, M. Portail, and G. Feuillet
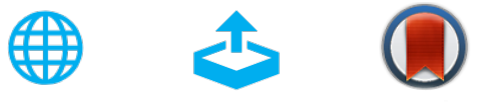

View Online

\section{ARTICLES YOU MAY BE INTERESTED IN}

Study of deep traps in AIGaN/GaN high-electron mobility transistors by electrical characterization and simulation

Journal of Applied Physics 125, 035702 (2019); https://doi.org/10.1063/1.5055926

The role of transient surface morphology on composition control in AIGaN layers and wells Applied Physics Letters 114, 031602 (2019); https://doi.org/10.1063/1.5063933

Methodology for the investigation of threading dislocations as a source of vertical leakage in AlGaN/GaN-HEMT heterostructures for power devices

Journal of Applied Physics 125, 095704 (2019); https://doi.org/10.1063/1.5065442

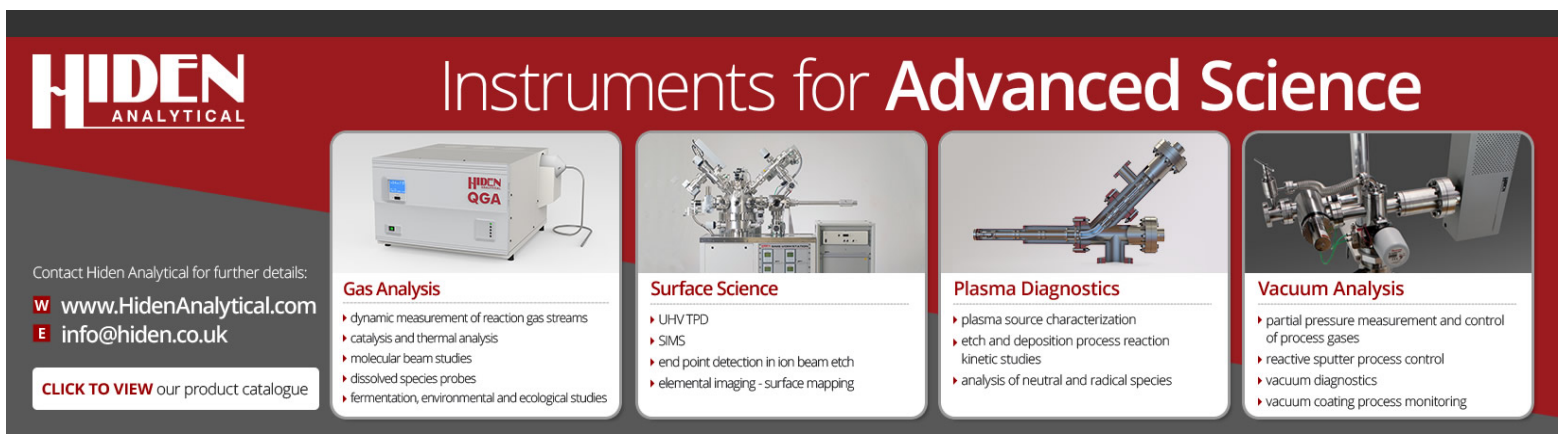




\title{
Semipolar (10-11) GaN growth on silicon-on- insulator substrates: Defect reduction and meltback etching suppression
}

\author{
Cite as: J. Appl. Phys. 125, 035703 (2019); doi: 10.1063/1.5067375 \\ Submitted: 17 October 2018 . Accepted: 1 January 2019. \\ Published Online: 16 January 2019
}

Rami Mantach, ${ }^{1,2}$ (ID P. Vennéguès, ${ }^{7}$ J. Zuniga Perez, ${ }^{7}$ (D) P. De Mierry, ${ }^{7}$ M. Leroux, ${ }^{7}$ M. Portail, ${ }^{7}$ and G. Feuillet ${ }^{2}$

\author{
AFFILIATIONS \\ ${ }^{7}$ CNRS, Université Côte d'Azur, CRHEA rue Bernard Grégory, 06560 Valbonne, France \\ ${ }^{2}$ CEA-LETI, MINATEC Campus, 17 rue des Martyrs, 38054 Grenoble, France
}

\begin{abstract}
We demonstrate the growth of almost strain-free (10-11) semipolar GaN on silicon-on-insulator (SOI) substrates, with no meltback etching and with a defect density strongly reduced compared to semipolar templates grown on patterned silicon substrates. This is carried out using SOI substrates with a very thin $(\sim 150 \mathrm{~nm}) 6^{\circ}$ off $(001)$ Si top layer. By resorting to very small nucleation (111) facets, revealed through chemical etching of the topmost thin Si layer, we are able to diminish significantly the overall dislocation density. Cathodoluminescence and scanning electron microscopy images at different stages of the growth illustrate how the defect density reduction operates and confirm the complete suppression of meltback etching over the whole 2 in. wafer. Low temperature photoluminescence and optical reflectivity indicate that complete strain relaxation is closely achieved $\left(\mathrm{D}^{0} \mathrm{X}\right.$ at $\left.3.473 \pm 0.001 \mathrm{eV}\right)$, compared to semipolar epilayers grown onto "bulk" silicon ( $\mathrm{D}^{0} \mathrm{X}$ at $\left.3.460 \mathrm{eV}\right)$. Thanks to this efficient strain relaxation, very thick layers, up to $9 \mu \mathrm{m}$, could be obtained crack-free.
\end{abstract}

Published under license by AIP Publishing. https://doi.org/10.1063/1.5067375

\section{INTRODUCTION}

Gallium nitride based materials are known for their exceptional luminescence efficiency in the visible range, leading the race for the lighting device revolution. ${ }^{1,2}$ However, GaN layers and heterostructures are usually grown along the polar direction (c-axis of the wurtzite structure), which results in internal polarization fields. ${ }^{3}$ These lead to an increase of the radiative lifetime $^{4}$ that, if combined with a large density of non-radiative defects, contributes to reducing the quantum efficiency and lifetime of devices. ${ }^{5}$

Semipolar and non-polar nitride materials have become a topic of interest because of their capability of reducing or even eliminating the effect of this internal polarization field ${ }^{6}$ and, on the other hand, possibly enhancing indium incorporation. Semipolar samples can be homo-epitaxially grown on semipolar GaN wafers resulting in very low dislocation densities, ${ }^{8}$ down to $10^{5}$ to $10^{4} \mathrm{~cm}^{-2}$, as demonstrated by Fujito et al. ${ }^{9}$ However, the semipolar wafers are still of very small sizes and rare, hence explaining the interest in heteroepitaxial semipolar GaN.

Several attempts for growing semipolar GaN directly on foreign substrates have been performed. However, they often resulted in poor quality epilayers with very large dislocation and basal stacking fault (BSF) densities $\left(10^{10} \mathrm{~cm}^{-2}\right.$ and $10^{5} \mathrm{~cm}^{-1}$, respectively). ${ }^{10}$ In situ low temperature (LT)-AlN interlayers have been used to block basal stacking faults (BSFs), but this method is not always efficient, particularly on semipolar orientations with high inclination angles with respect to the c-axis. ${ }^{11}$ Hence, there is a clear interest in finding a way that leads to low dislocation and BSF densities in heteroepitaxial semipolar layers.

Honda et al. were the first to grow semipolar (10-11) GaN on patterned substrates. In their approach, the growth takes place on inclined $v$-shaped (111) facets etched on the silicon substrate ${ }^{12}$ the inclination of which determines the orientation of the GaN semipolar epilayer grown thereon. Accordingly, various semipolar orientations were achieved, including (11-22) GaN layer on the $r$-plane patterned sapphire substrate (PSS), ${ }^{13,14}$ (11-22) GaN on the patterned (113) Si substrate, ${ }^{15}$ and (20-21) GaN on the (114) $1^{\circ}$ off patterned Si substrate. ${ }^{16}$

In the case of sapphire substrates, several methods have been implemented to reduce the dislocation density in the semipolar GaN layers; in particular, the so-called 3-steps 
growth on $\mathrm{PSS}^{13}$ results in a record threading dislocation density in the mid $-10^{7} \mathrm{~cm}^{2}$ range and a BSF density of around $100 \mathrm{~cm}^{-1}$, establishing the current state of the art. (20-21) semipolar GaN and InGaN quantum well were also demonstrated on similarly patterned sapphire substrates. ${ }^{17}$ Recently, semipolar (11-22) $550 \mathrm{~nm}$ yellow/green ${ }^{18}$ and $444 \mathrm{~nm}$ blue InGaN light-emitting diodes (LEDs) ${ }^{19}$ and semipolar (20-21) GaN and InGaN LEDs ${ }^{20}$ were demonstrated on sapphire templates using this defect filtering method.

Growth is more challenging on patterned silicon substrates, among other reasons due to the meltback etching problem: a reaction between $\mathrm{Si}$ and $\mathrm{Ga}$ that initiates during growth and results in a violent outspread of matter causing complete destruction of the sample surface. ${ }^{21} \mathrm{Yu}$ et al. succeeded to grow semipolar (20-21) GaN on stripe patterned (113) Si with no meltback etching. ${ }^{15}$ Khoury et al. eliminated the reaction by adding a small amount of $\mathrm{N}_{2}$ to the carrier gas during the growth, but at the expense of negatively affecting the coalesced GaN quality by increasing the number of generated structural defects. The state of the art for the dislocation density in semipolar GaN grown on patterned silicon substrate is $2.7 \times 10^{8} \mathrm{~cm}^{-2}$. 22

In the work reported here, we demonstrate the growth of (10-11) GaN layers grown on patterned (001) $6^{\circ}$ off silicon-on-insulator (SOI) substrates that allow to further reduce the dislocation density and eliminate meltback etching. Following $\mathrm{KOH}$ etching of the thin enough (see later) top Si layer, growth takes place on very small $\mathrm{Si}$ inclined facets. This is the key to reducing the defect density, which relies on dislocation bending in pyramidal grains as explained by Vennéguès et al. ${ }^{23}$ The combination of small nucleation facets and, therefore, of small pyramids results in the confinement of the emerging dislocations in a very narrow zone of the layer surface. These regions are surrounded by almost dislocation free areas. ${ }^{13,23}$ Both the width of the region containing the threading dislocations and the number of nucleated dislocations depend on the facet size, pinpointing the importance of SOI substrates. We also show that another important advantage related to using SOI substrates and small growth facets is the elimination of the meltback etching, aided by the strong reduction of the interaction surface between $\mathrm{Ga}$ and $\mathrm{Si}$.

\section{EXPERIMENTAL METHOD}

Unlike standard patterned bulk Si substrate, our substrate is an SOI wafer composed of a top (001) $6^{\circ}$ off Si layer whose thickness determines the size of the Si growth facet. For this work, the top Si layer is $150 \mathrm{~nm}$ thick, while the $\mathrm{SiO}_{2}$ layer, present between the two Si layers, has a thickness of $500 \mathrm{~nm}$. The bottom Si substrate is $<001>$ oriented with a thickness of $500 \mu \mathrm{m}$.

\section{Patterning}

The successive patterning steps are depicted in Fig. 1. The first step is the deposition of a $50 \mathrm{~nm}$ thick $\mathrm{SiO}_{2}$ mask on the top Si using ion beam sputtering (IBS) (Fig. 1, step 1). The patterned bands, lying along the [110] Si direction, are then defined using standard photolithography with a $5 \mu \mathrm{m}$ period (2.5 $\mu \mathrm{m}$ opening and $2.5 \mu \mathrm{m}$ mask) (Fig. 1, step 2). The apertures of the $\mathrm{SiO}_{2}$ masks are dry etched using an inductively coupled plasma (Fig. 1, step 3). The sample is then cleaned for $30 \mathrm{~s}$ in a buffered oxide etch (BOE) to eliminate the native oxide, which often prevents $\mathrm{KOH}$ from etching silicon.

The substrate is then exposed to wet etching using $\mathrm{KOH}$, revealing (111) and (111) opposite facets. In our case, these facets have an inclination of $48^{\circ}$ and $60^{\circ}$, respectively, with respect to the $6^{\circ}$ off (001) top Si surface (Fig. 1, step 4). If we would have used patterned Si substrates instead of SOI, etching would have stopped automatically once a complete "V" shape groove was formed. This would have resulted in two large $\left(2-3 \mu \mathrm{m}\right.$ wide) opposing $\{111\}$ Si facets, ${ }^{16}$ whose size would have determined the defect density. Besides, the homogeneity of $\mathrm{KOH}$ etching was reported to be poor for bulk Si substrates; ${ }^{22}$ this is due to the random initiation of $\mathrm{KOH}$ etching because of the thickness non-uniformity of the native silicon oxide layer on the silicon surface. Therefore, locally retarded etching may yield different Si facet sizes, making coalescence of neighboring bands/grains random. To achieve a perfect uniformity of the facet sizes over the whole substrate surface, we used SOI substrates. Indeed, the top Si layer can be totally etched, the $\mathrm{SiO}_{2}$ layer just below acting as a perfect etch-stop. The thickness homogeneity for the top $\mathrm{Si}$ layer of the SOI substrate was measured by reflectometry and found to be better than $\pm 2 \%$ on a 2 -in. wafer, therefore yielding very homogeneous facet heights.

Subsequently, a $50 \mathrm{~nm}$ thick AlN layer is grown (Fig. 1, step 5) at $1210{ }^{\circ} \mathrm{C}$ using a Metal-Organic Vapor Phase Epitaxy (MOVPE) vertical close coupled shower head reactor. ${ }^{24}$ Growing AlN on the Si surface is a prerequisite to avoid meltback etching from occurring through the direct contact between $\mathrm{Ga}$ and $\mathrm{Si}$. The used metal precursor is trimethylaluminum and the temperature is measured using an in situ thermocouple below the susceptor pockets where the substrates are placed.

The final step consists in the inclined deposition of $\mathrm{SiO}_{2}$ (Fig. 1, step 6) using IBS. This is done in order to cover the top surfaces but also the $\{111\} 48^{\circ}$ inclined facets while avoiding any deposition on the $60^{\circ}$ inclined facet, where GaN growth needs to be initiated. Figure 2 is a scanning electron microscope (SEM) cross-sectional image of the final patterned SOI surface (the $50 \mathrm{~nm}$-thick AlN seed layer is hard to visualize at such a magnification).

\section{Growth}

The same MOVPE reactor as the one for AlN deposition was used for the selective growth of GaN. The precursors were trimethylgallium (TMGa) and ammonia $\left(\mathrm{NH}_{3}\right)$. The growth process was divided into two steps: the first one is the growth of $\mathrm{GaN}$ pyramids at $1090^{\circ} \mathrm{C}$, high pressure (300 Torr), and a $\mathrm{V} / \mathrm{III}$ ratio of 1500 . In a second step, in order to achieve coalescence, growth is continued at the same temperature but lower pressure (100 Torr) and lower V/III ratio (650). It is 


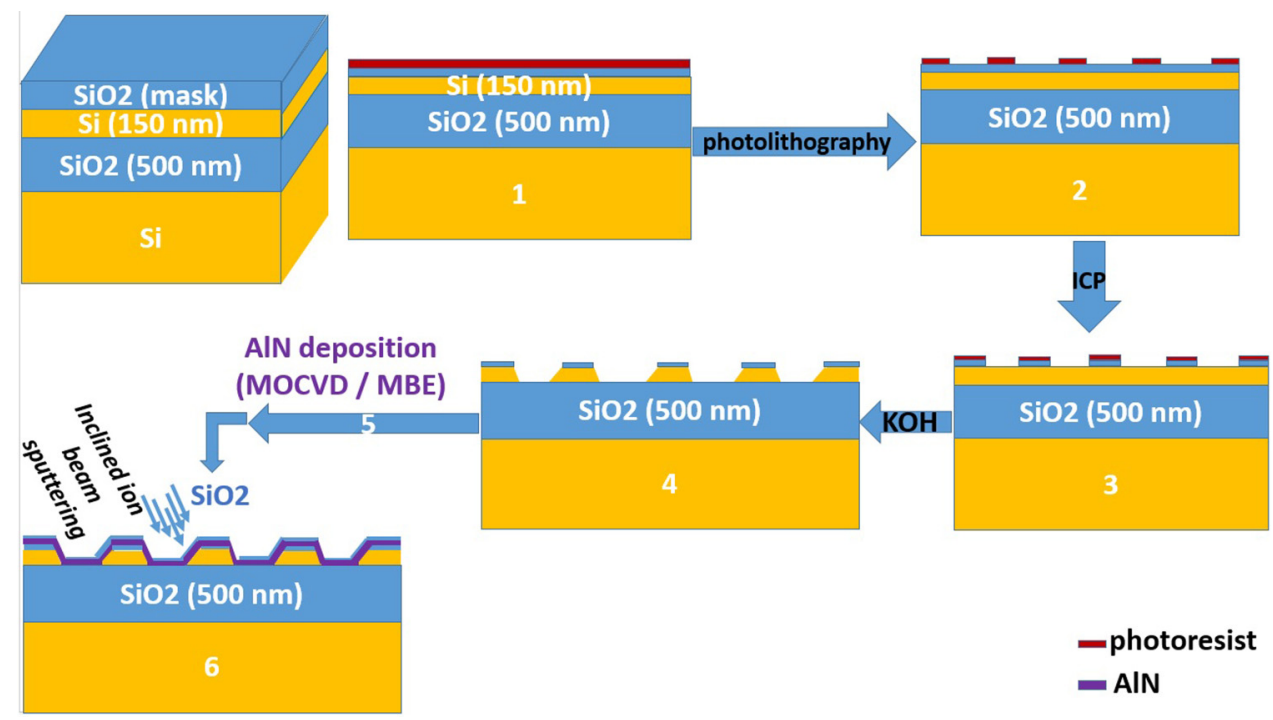

FIG. 1. Patterning steps of SOI. 1, photoresist deposition; 2, photolithography; $3, \mathrm{SiO}_{2}$ dry etching; 4 , SOI wet etching; 5 , AIN deposition; 6 , $\mathrm{SiO}_{2}$ deposition using an inclined beam sputtering.

worth mentioning that using the same growth parameters as for the GaN pyramids on large Si growth facets (as obtained when using the bulk Si substrate) results in parasitic growth: indeed, GaN islands are nucleated on the entire surface (i.e., also on the $\mathrm{SiO}_{2}$ mask) and lead to the formation of incomplete GaN pyramids on the growth facets. Therefore, pressure, TMGa, and $\mathrm{NH}_{3}$ fluxes should be adjusted for the nucleation and growth onto small facets. This can be understood by considering that the growth on the facet is related to two adatoms fluxes: one from direct deposition and the other from species diffusion on the mask from either side of the growth facet. If the growth conditions employed for small facets are identical to those used for large facets (TMGa fluxes of 2200 instead of $1600 \mu \mathrm{mol} / \mathrm{min})$, the reservoir of species available for growth is too high, eventually leading to parasitic deposition on the $\mathrm{SiO}_{2}$ mask from either side of the small facet. This explains the necessary growth adjustments when going from large to small facet sizes.

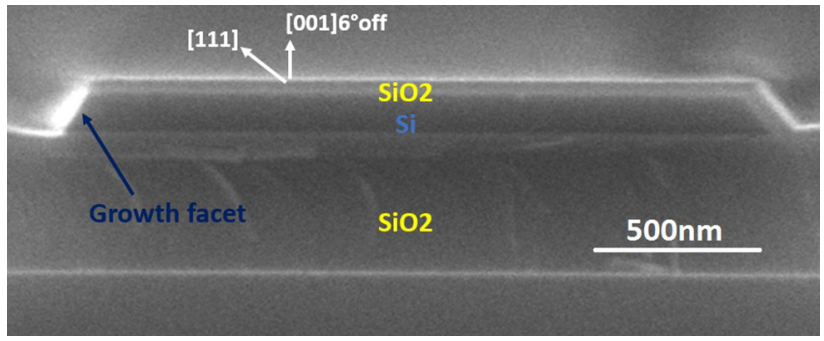

FIG. 2. A cross-sectional SEM image for the SOI substrate after patterning. Growth facet and $\mathrm{SiO}_{2}$ mask are shown.

\section{RESULTS AND DISCUSSION}

Figure 3(a) shows the formation of perfect GaN pyramids after $500 \mathrm{~s}$ of growth at 300 Torr using $3.5 \mathrm{slm} \mathrm{NH}_{3}$. The aim is to create a pyramid at the beginning of growth in order to bend all dislocations nucleating on the inclined Si facets. ${ }^{13,19}$ This growth stage is referred to as stage A. Continuing growth under the same condition for $1500 \mathrm{~s}$ leads to the formation of small c facets as shown in Fig. 3(b) (stage B). At this particular moment, the growth parameters are changed in order to favor the growth in the $+c$ direction for reaching coalescence: the pressure is decreased to 100 Torr and the ammonia flow to $1.5 \mathrm{slm}$, keeping the TMGa flow constant.

Appropriately splitting the growth time between the 2 steps enables fast coalescence for the formation of a continuous GaN layer. Figures 3(c) and 3(d) show a coalesced layer for a growth duration of $8000 \mathrm{~s}$ (overall time) which resulted in a $7 \mu \mathrm{m}$-thick crack free GaN (10-11) epilayer (stage C).

The first important result is that, contrary to the case where bulk Si substrates are used for growing semipolar $\mathrm{GaN}$, no meltback etching was detected on the surface of the sample. This is achieved without having to use methods such as, for instance, that reported in Ref. 21, where an $\mathrm{N}_{2}$ flow was added to the $\mathrm{H}_{2}$ carrier gas. In the case of c-oriented twodimensional GaN growth, the use of an AlN buffer layer is sufficient for avoiding melt back etching, without having to resort to reducing the substrate footprint. For semipolar nitrides, things are a lot different since patterning the Si substrate seems to have a great influence on melt-back etching. The reason for the different behaviors is not clear at present. But one may assume that it can be related to the way the (111) $\mathrm{Si}$ surface is prepared. Indeed, $\mathrm{KOH}$ etching used here for defining $\{111\}$ facets may leave either a much more reactive 

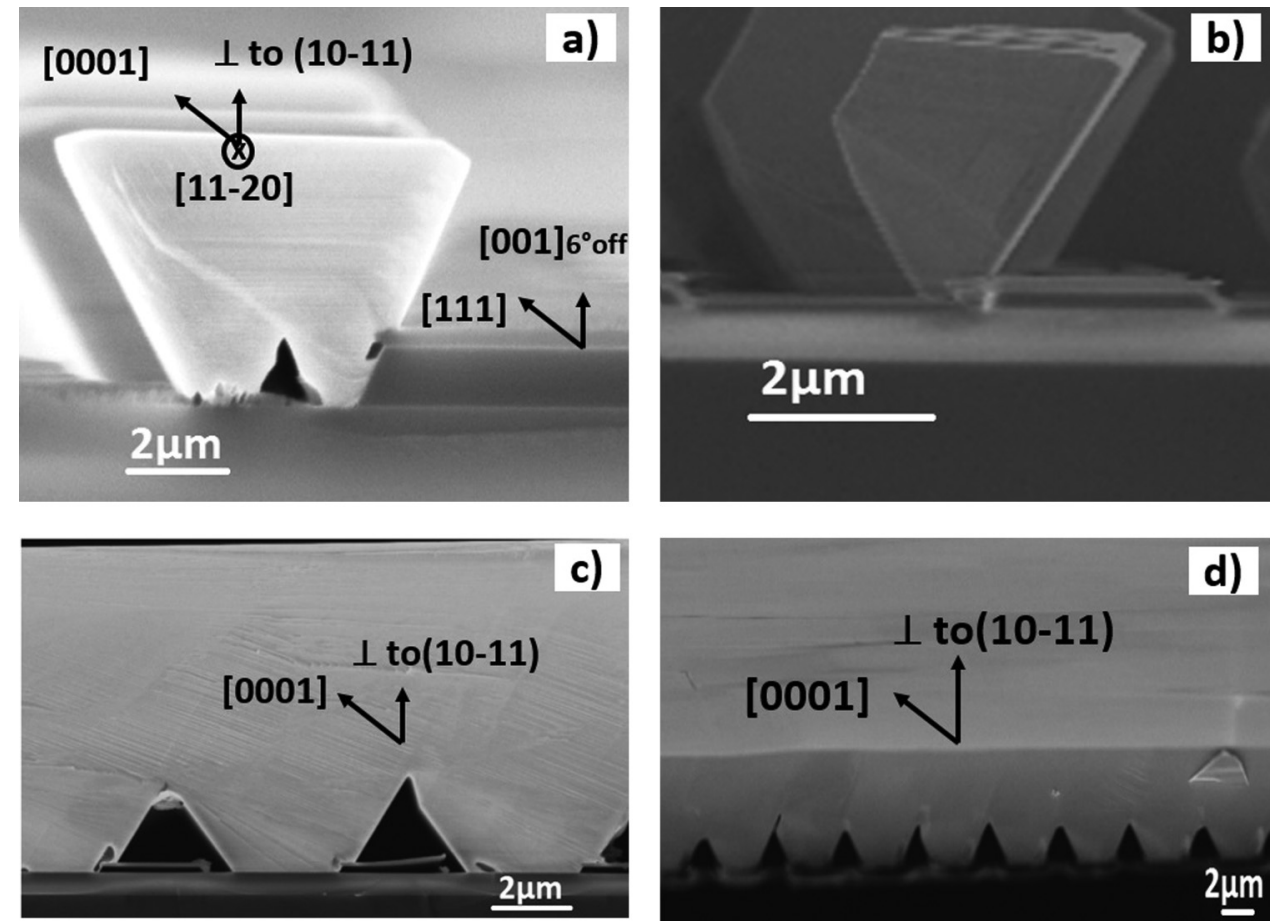

FIG. 3. Cross-sectional SEM images showing the evolution of pyramids that form during growth after (a) $500 \mathrm{~s}$, (b) $1500 \mathrm{~s}$, [(c) and (d)] $8000 \mathrm{~s}$.

surface with respect to $\mathrm{Ga} / \mathrm{Si}$ interactions than in the case of surfaces obtained by chemical-mechanical polishing (CMP); furthermore, $\mathrm{KOH}$ etching may leave surface imperfections (steps, macrosteps, impurity clusters) inducing AlN thickness irregularities, through which Ga diffusion may be favored. If this were the case, reducing the $\mathrm{Si}$ footprint for growth reduces the probability for encountering such surface imperfections. A second reason might be related to the different etching times required to reveal the growth facets, which are much shorter in the current work. Indeed, since at roomtemperature the $(100) /(111)$ etching rate ratio amounts to about 100 , if during the $\mathrm{KOH}$ etching the $\{111\}$ facets form steps, the anisotropic etching ratio might lead to the formation of surface pits that, once again, might enhance the meltback etching probability. Thus, in the present case of semipolar growth on etched $\{111\}$ Si facets, the AlN layer alone cannot do the job by itself, as shown in Refs. 16 and 21, where meltback occurred despite the use of an AlN buffer layer. Hence, the advantage of using a smaller substrate footprint.

Cathodoluminescence (CL) was carried out in a JEOL $7000 \mathrm{~F}$ scanning electron microscope (SEM) equipped with a Gatan Mono CL4 module. CL images were used in order to locate the defects, mainly dislocations and basal stacking faults (BSFs) in the layers at the different stages of growth (A: pyramid nucleation, B: pyramid lateral extension leading to continuous stripes, and $\mathrm{C}$ : completely coalesced layer). Threading dislocations are non-radiative centers and as such appear as dark spots on panchromatic CL images. Most measurements have been done at $77 \mathrm{~K}$, allowing us to detect the presence of BSFs, which act as radiative centers with associated levels in the gap, relatively close to the band edge, hence being difficult to detect at room temperature (RT). ${ }^{25}$ Figure 4 shows the $77 \mathrm{~K}$ panchromatic CL images of samples at the three stages of growth (A, B, and C). After stage A [Fig. 4(a)], corresponding to pyramid nucleation, a non-radiative spotty line, presumably associated to dislocations coming from the growth facet, can be seen (red arrows) and appears over a very limited width. Within the bands in Figs. 4(a) and 4(b), bright and dark regions with triangular shape are present as well. These are related to the overlapping of adjacent pyramids that, upon coalescence in the direction of the bands, have left some underlying voids between them [as observed in cross-sectional SEM images; see Figs. 3(c) and 3(d)]. Dislocations are not seen anywhere else. After stage B [Fig. 4(b)], complete GaN stripes were formed and no additional dislocations are seen. It is noteworthy that BSFs are not created unless growth parameters are changed. Indeed, they start to be created while growing in the $-c$ direction before complete coalescence occurs. Besides, the coalescence of the bands from adjacent stripes is accompanied too by the occurrence of basal stacking faults, as evidenced from their luminescence [Fig. 4(c)].

Most interestingly, the CL images in Fig. 5(a), taken from the top of the layers, reveal that the width of the high 

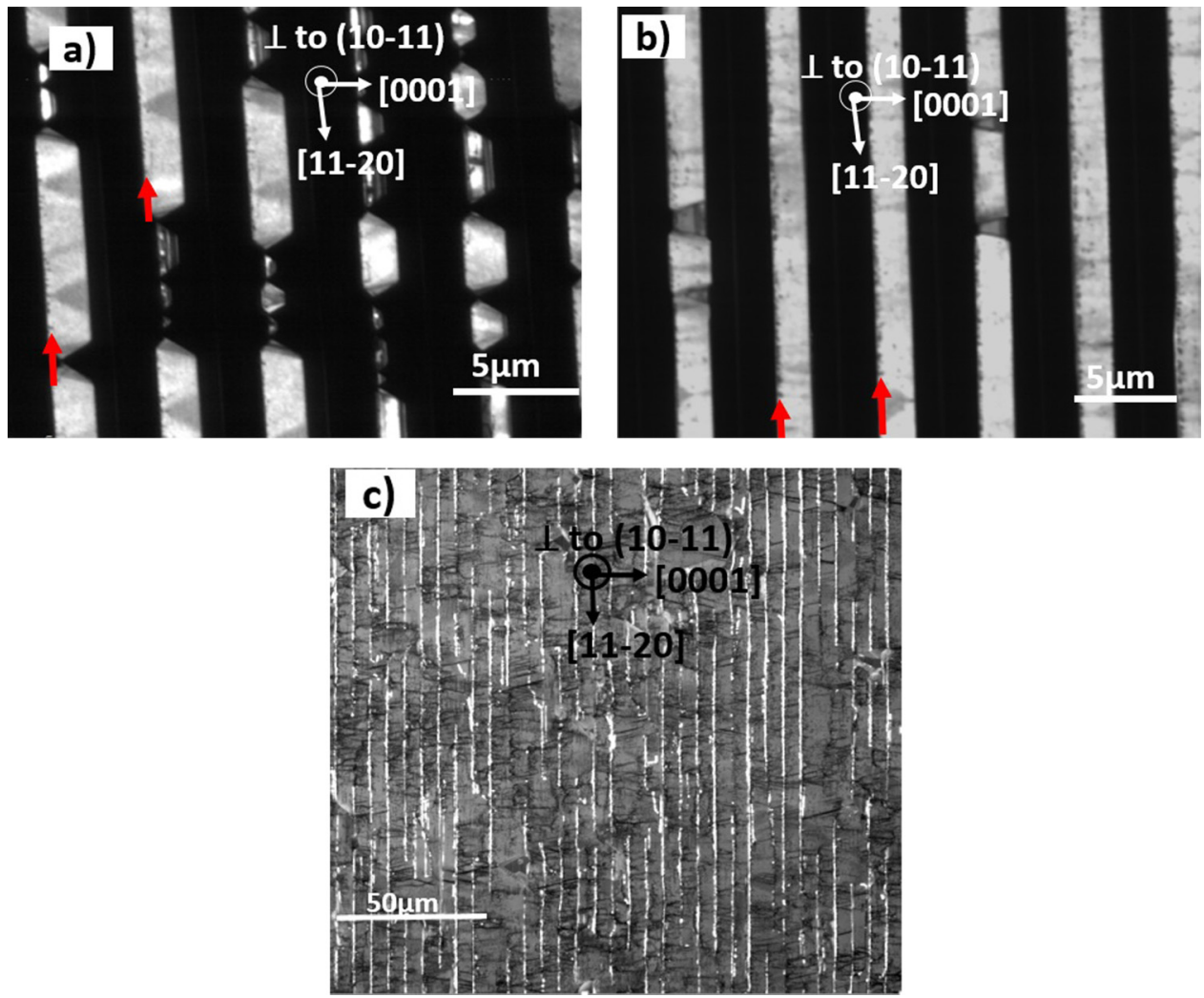

FIG. 4. Cathodoluminescence images (77 K) for the pyramids nucleation (a), for the pyramids coalescence (b), and for the strips coalescence (c). Dislocations are shown as black dots and lines; BSFs are the bright lines.

dislocation density regions is limited to $200-300 \mathrm{~nm}$ in fully coalesced layers [Fig. 5(a)]. For comparison, in the case of large facets [Fig. 5(b)], ${ }^{22}$ as obtained for semipolar growth on bulk $7^{\circ}$ off (001) Si substrates, the typical width of the emerging dislocation area is around $4 \mu \mathrm{m}$ for $\{111\} 4-5 \mu \mathrm{m}$ large $\mathrm{Si}$ facet. This highlights the second advantage brought about by the use of patterned thin SOI in terms of dislocation reduction. From simple geometrical considerations, one can deduce the width of the dislocation zone at the surface of the semipolar sample. As already discussed, dislocations originate at the $\mathrm{Si} / \mathrm{AlN}$ interface and propagate along the c direction. Upon intersecting one of the GaN pyramid facets, these dislocations bend in the c-plane either upward or downward, in order to minimize their elastic energy (Fig. 6). Therefore, emerging dislocations are confined in a region of the surface (of width W) situated above the nucleation facet. The last dislocation to be bent is the one closest to the pyramid apex. This determines the width $\mathrm{W}$ of the large density dislocation region on the surface, which is simply the projection of the pyramid facet on the layer's surface. In our case, because the facet is inclined at $60^{\circ}$ to the surface, we infer a width of the dislocated zone W $=\mathrm{H} / \sin 60$, with $\mathrm{H}$ being the thickness of the top Si layer of the SOI. $H=150 \mathrm{~nm}$, hence $\mathrm{W} \sim 175 \mathrm{~nm}$ in fairly good agreement with the experimental width. Outside this narrow dislocated region, the dislocation density crossing the rest of the surface is approximatively $4 \times 10^{7} \mathrm{~cm}^{-2}$. All in all, and taking the bent dislocations into account, the dislocation density was calculated using room temperature CL images where BSFs are not seen and was found to be about $10^{8} \mathrm{~cm}^{-2}$.

Other dark lines inclined at $75^{\circ}$ to the $\mathrm{e}^{11-20}$ direction can also be seen, as evidenced in Fig. 5(a), corresponding to dislocations, or clusters of dislocations, appearing only after coalescence has occurred between neighboring stripes. These dislocations are presumably generated by the relaxation of the strained GaN layer through dislocation glide in the prismatic $m$-plane, as expected in semipolar GaN. ${ }^{26}$

Now, concerning BSFs, they start forming when the GaN pyramids begin over-growing the $\mathrm{SiO}_{2}$ mask in the -c direction. Growth rate in the $+c$ direction is much faster than in the $-c$ direction, the ratio being as large as 7 during epitaxial-lateralovergrowth experiments. ${ }^{27}$ Indeed, it is believed that lower growth rates result in higher impurity incorporation, leading to enhanced formation of stacking faults, hence higher BSFs density in the $-c$ direction compared to the $+c$ one. ${ }^{28}$ The panchromatic images in Figs. 4(a) and 4(b) show that no BSFs are formed before changing the growth parameters in order to 

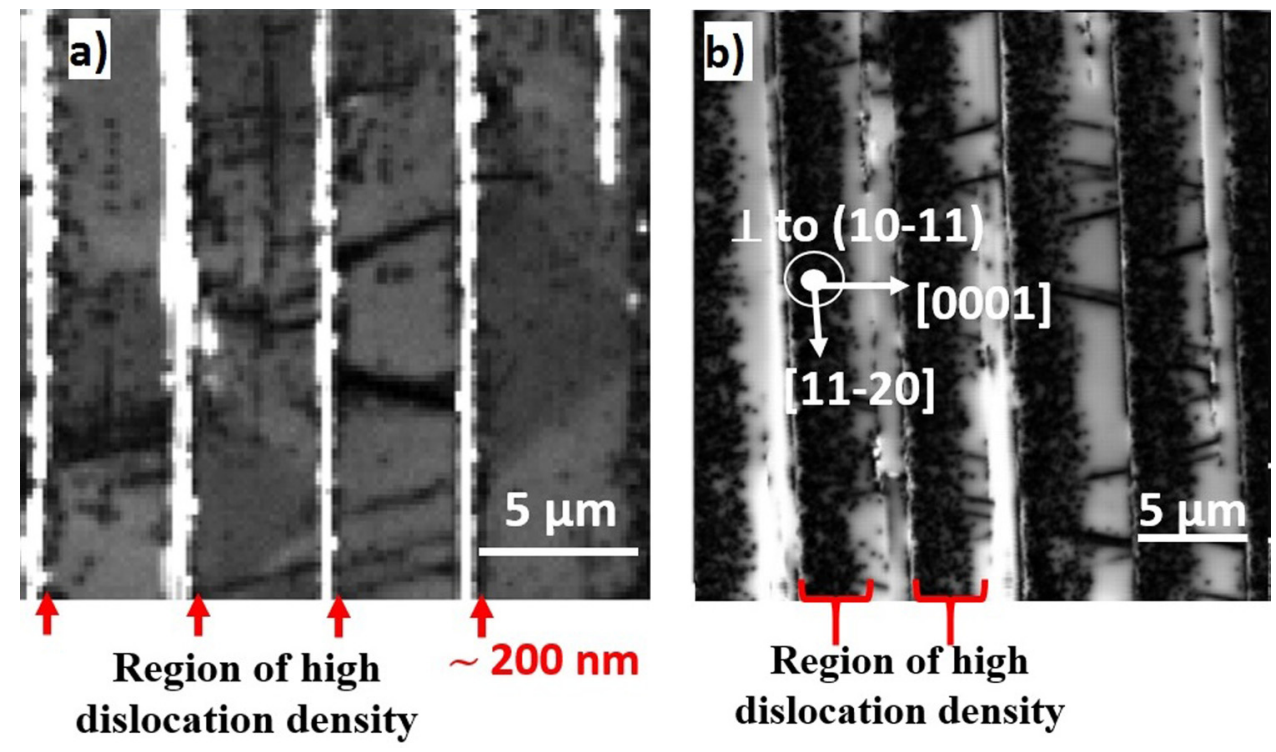

FIG. 5. High dislocation density region in the case of $\mathrm{GaN}$ on SOI $(77 \mathrm{~K})$ (a) and on Si bulk substrates (300 K) (b).

favor the coalescence of the stripes. This is mainly due to the fact that the growth parameters employed during the first growth step fully favor growth along $+c$ and, thus, GaN does not grow in the $-c$ direction even if the pyramids size is much larger than the Si facet size. On the other hand, the growth conditions in the second step favor two-dimensional growth and result in some -c growth. In Fig. 4(c), where the growth is complete, BSF (bright lines) and dislocations (black lines) appear with the periodicity of the underlying Si facets. The density of BSFs was estimated from the CL images. Our counting procedure consists in acquiring CL images $10 \mu \mathrm{m} \times 10 \mu \mathrm{m}$ large, summing the length of bright lines and dividing it by the image surface $\left(100 \mu \mathrm{m}^{2}\right)$. This gives a density of bright lines in the order of $3-4 \times 10^{3} \mathrm{~cm}^{-1}$. Assuming a density of $10 \mathrm{BSFs} /$ $100 \mathrm{~nm}$ in the regions of bright lines, as previously measured by TEM, ${ }^{29}$ we can estimate a BSFs density in the low $10^{5} \mathrm{~cm}^{-1}$ (high $10^{4} \mathrm{~cm}^{-1}$ ). Contrarily, for semipolar GaN grown on bulk $\mathrm{Si}$ substrates employing a one-step growth, GaN pyramids are
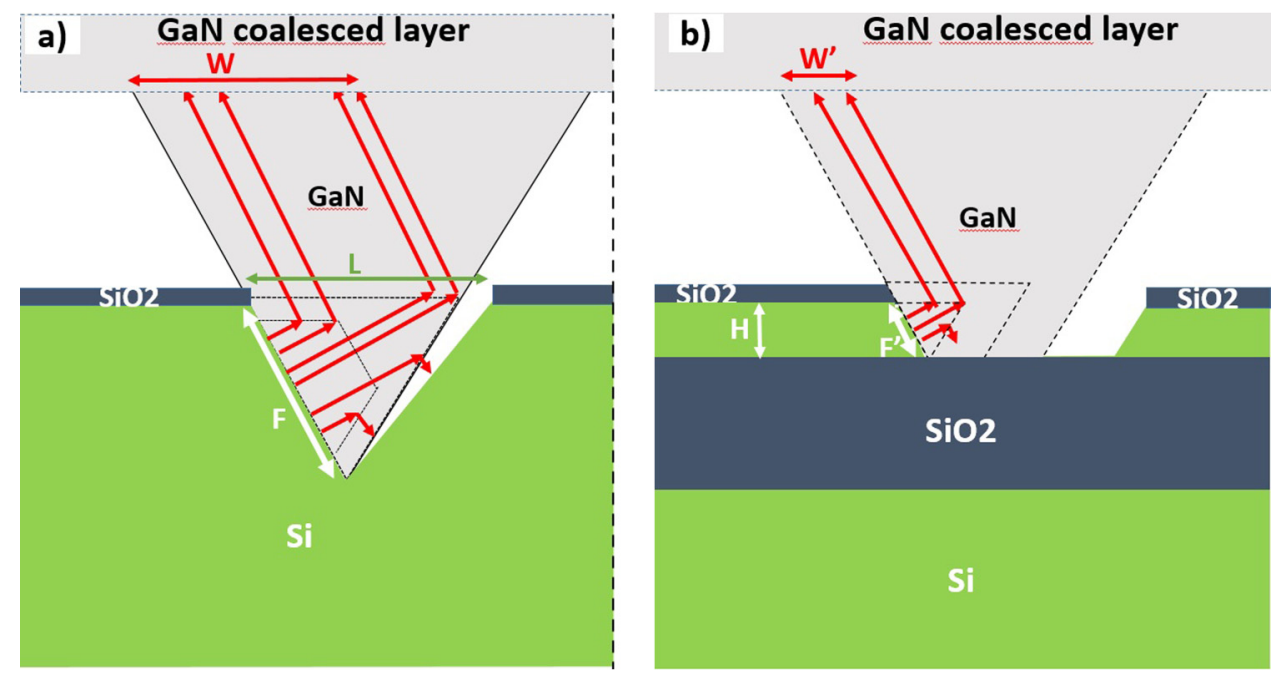

FIG. 6. Scheme showing the dislocation bending phenomenon and the high dislocation region "W." 
free to overgrow the $\mathrm{SiO}_{2}$ mask in the -c direction, leading to the formation of a larger number of BSFs. Our study consists in growing $\mathrm{GaN}$ in two growth steps, allowing for a better control of growth in the $-c$ direction. Indeed and as evidenced in Fig. 3, the BSFs are absent in the individual pyramids and in the bands before changing the growth parameters in order to achieve coalescence. The photoluminescence (PL) spectra (shown later in Fig. 7) illustrate the difference between the BSF related intensity of semipolar GaN on bulk $\mathrm{Si}$ and SOI substrates, which is coherent with the reduced density of BSFs extracted from our CL study.

Photoluminescence (PL) was used not only to probe the optical quality of the semipolar (10-11) epilayers but also for investigating defects, essentially BSFs. PL measurements were carried out at $15 \mathrm{~K}$. Figure 7 compares the PL spectra of two different semipolar samples with the same (10-11) orientation: on patterned SOI (black line) and on patterned bulk Si (red line). The PL bands near $3.405 \mathrm{eV}$ involve $\mathrm{I}_{1}$ basal stacking faults. Hence, Fig. 7 evidences a strong reduction of their relative contributions in the case of the sample grown on SOI, corresponding to a reduced density. This is consistent with the CL observations discussed above. As it is well known, the position of the near band edge luminescence is closely related to the strain. While the donor bound exciton $\left(\mathrm{D}^{0} \mathrm{X}\right)$ of semipolar $\mathrm{GaN}$ on bulk $\mathrm{Si}$ emits at $3.460 \mathrm{eV}$, indicative of a tensile strain (in relaxed GaN, the energy of excitons bound to the residual donors $\mathrm{Si}$ or $\mathrm{O}$ is $3.471-3.472 \mathrm{eV}$ ), in the sample on SOI its energy is $3.473 \mathrm{eV}$. Rather than indicating a compressive strain, this emission energy points toward the presence of a second emission contribution, which corresponds to freeexciton emission. Indeed, $15 \mathrm{~K}$ reflectivity spectrum, also shown in Fig. 7 (blue line), shows a free excitonic feature at $3.475 \pm 0.001 \mathrm{eV}$, confirming a much lower tensile strain than on Si substrates, but not a compressive strain (the energy of A

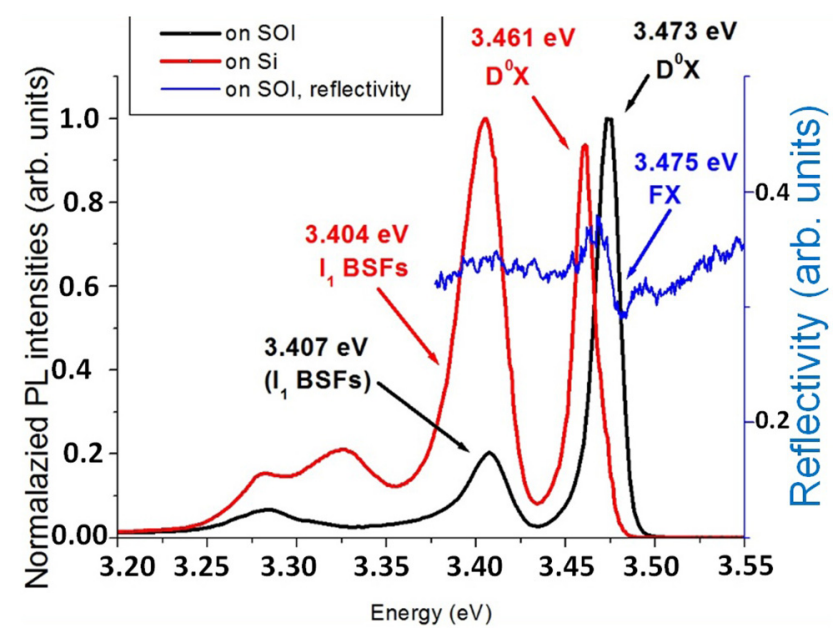

FIG. 7. A photoluminescence spectrum (10 K) for $\mathrm{GaN}$ on SOI (black line) and on Si bulk (red line); and finally the blue line is the reflectivity of GaN at $12 \mathrm{~K}$. excitons is $3.477-3.478 \mathrm{eV}$ in relaxed $\mathrm{GaN})$. Interestingly, as was apparent in Fig. 5(a), a crack can be detected, synonymous of the relaxation of a residual tensile stress in the layers: the semipolar layers are very close to full relaxation but still subject to a very small residual tensile stress explaining why there are very few cracks, even for $8-9 \mu \mathrm{m}$ GaN thicknesses. In fact, most of the surface is crack-free, as seen for instance in Figs. 3(d) and 4(c).

The mechanism beyond this almost full relaxation is not well understood yet. GaN pyramids and stripes (i.e., before coalescence) may relax elastically due to available free surfaces at sufficiently close distances (at most $2.5 \mu \mathrm{m}$ away). For coalesced films, one hypothesis would be that the stress is transferred from the thick $(8-9 \mu \mathrm{m}) \mathrm{GaN}$ layer to the thin $(150 \mathrm{~nm})$ and non-continuous top Si layer, which should find itself deformed. We are currently working to assess this hypothesis. Still, one should note that the exciton energy reveals that there exists a residual, albeit very small, tensile strain in the coalesced layer. This very small tensile stress explains why there are almost no cracks, even for $8-9 \mu \mathrm{m}$ $\mathrm{GaN}$ thicknesses. A study of strain evolution as growth proceeds will be reported later.

The absence of stress in semipolar heterostructures when grown on SOI is of course very important, as it will allow us to design, for instance, optimal LED structures not requiring strain-compensating layers.

\section{CONCLUSION}

We have demonstrated the growth of (10-11) GaN on SOI substrates on which we have fabricated nanometric facets, $150 \mathrm{~nm}$ wide. This specific substrate structure and this thin $\mathrm{Si}$ top layer have been used to reduce the dislocation density in $\mathrm{GaN}$, given that it is essentially determined by the footprint of GaN on the substrate. Melt-back etching has also been suppressed taking advantage of the SOI structure, which enables to easily avoid the direct interaction between $\mathrm{Ga}$ and $\mathrm{Si}$ since, once again, the GaN footprint on the $\mathrm{Si}$ material is strongly reduced compared to a standard growth. BSFs creation and evolution have also been studied by CL and PL measurements that show no BSFs at the early growth stages, during which pyramids grow only along the $+c$ direction. Cathodoluminescence images showed a drastic reduction of dislocation density after being bent and regrouped in a very narrow region compared to the standard approach using large nucleation facets. Finally, photoluminescence spectra show that the GaN layers grown on SOI are almost strainfree, paving the way for growing high-quality thick semipolar layers to be used as templates for light-emitting devices and suggesting the possibility of employing patterned SOI as a compliant substrate.

\section{ACKNOWLEDGMENTS}

The authors would like to thank GANEX (ANR-11-LABEX0014) and CEA for funding this work. GANEX belongs to the public funded "Investissement d'Avenir" program managed by French ANR agency. We also thank Michel Khoury from 
UCSB (University California Santa Barbara) and Helge Haas (CEA, LETI) for enlightening discussions and essential suggestions.

\section{REFERENCES}

${ }^{\mathbf{1}}$ F. A. Ponce and D. P. Bour, Nature 386, 351 (1997).

${ }^{2}$ E. F. Schubert and J. K. Kim, Science 308, 1274 (2005).

${ }^{3}$ F. Bernardini and V. Fiorentini, Phys. Rev. B 56, R10024 (1997)

${ }^{4}$ R. Langer, J. Simon, V. Ortiz, N. T. Pelekanos, A. Barski, R. André, and M. Godlewski, Appl. Phys. Lett. 74, 3827 (1999).

${ }^{5}$ P. Waltereit, O. Brandt, A. Trampert, H. T. Grahn, J. Menniger, M. Ramsteiner, M. Reiche, and K. H. Ploog, Nature 406, 865 (2000).

${ }^{6}$ V. W. Scarola, K. Park, and J. K. Jain, Nature 406, 863 (2000).

${ }^{7}$ J. E. Northrup, J. Neugebauer. Phys. Rev. B 60, R8473 (1999).

${ }^{8}$ T. Hashimoto, F. Wu, J. S. Speck, and S. Nakamura, Nat. Mater. 6, 568 (2007).

${ }^{9}$ K. Fujito, K. Kiyomi, T. Mochizuki, H. Oota, H. Namita, S. Nagao, and I. Fujimura, Phys. Status Solidi A 205, 1056 (2008).

${ }^{10}$ T. J. Baker, B. A. Haskell, F. Wu, J. S. Speck, and S. Nakamura, Jpn. J. Appl. Phys. 45, L154 (2006).

${ }^{11}$ A. Dadgar, R. Ravash, P. Veit, G. Schmidt, M. Müller, A. Dempewolf, F. Bertram, M. Wieneke, J. Christen, and A. Krost, Appl. Phys. Lett. 99, 021905 (2011).

${ }^{12}$ Y. Honda, N. Kameshiro, M. Yamaguchi, and N. Sawaki, J. Cryst. Growth 242, 82 (2002).

${ }^{13}$ F. Tendille, P. De Mierry, P. Vennéguès, S. Chenot, and M. Teisseire, J. Cryst. Growth 404, 177 (2014).

${ }^{14}$ F. Tendille, M. Hugues, P. Vennéguès, M. Teisseire, and P. De Mierry, Semicond. Sci. Technol. 30, 065001 (2015).
${ }^{15}$ X. Yu, Y. Hou, S. Shen, J. Bai, Y. Gong, Y. Zhang, and T. Wang, Phys. Status Solidi C 13, 190 (2016).

${ }^{16}$ M. Khoury, M. Leroux, M. Nemoz, G. Feuillet, J. Zúñiga-Pérez, and P. Vennéguès, J. Cryst. Growth 419, 88 (2015).

${ }^{17}$ B. Leung, D. Wang, Y.-S. Kuo, K. Xiong, J. Song, D. Chen, S. H. Park, S. Y. Hong, J. W. Choi, and J. Han, Appl. Phys. Lett. 104, 262105 (2014).

${ }^{18} \mathrm{H}$. Li, M. Khoury, B. Bonef, A. I. Alhassan, A. J. Mughal, E. Azimah, M. E. A. Samsudin, P. De Mierry, S. Nakamura, J. Speck, and S. P. DenBaars, ACS Appl. Mater. Interfaces 9, 36417 (2017).

${ }^{19}$ M. Khoury, H. Li, L. Y. Kuritzky, A. J. Mughal, P. DeMierry, S. Nakamura, J. S. Speck, and S. P. DenBaars, Appl. Phys. Express 10, 106501 (2017).

${ }^{20}$ J. Song, J. Choi, K. Xiong, Y. Xie, J. J. Cha, and J. Han, ACS Appl. Mater. Interfaces 9, 14088 (2017).

${ }^{\mathbf{2 1}} \mathrm{M}$. Khoury, O. Tottereau, G. Feuillet, P. Vennéguès, and J. Zúñiga-Pérez, J. Appl. Phys. 122, 105108 (2017).

${ }^{22}$ M. Khoury, P. Vennéguès, M. Leroux, V. Delaye, G. Feuillet, and J. Zúñiga-Pérez, J. Phys. D Appl. Phys. 49, 475104 (2016).

${ }^{23}$ P. Vennéguès, B. Beaumont, V. Bousquet, M. Vaille, and P. Gibart, J. Appl. Phys. 87, 4175 (2000).

${ }^{24}$ T. Tanikawa, T. Hikosaka, Y. Honda, M. Yamaguchi, and N. Sawaki, Phys. Status Solidi C 5, 2966 (2008).

${ }^{\mathbf{2 5}}$ T. Gühne, Z. Bougrioua, S. Laügt, M. Nemoz, P. Vennéguès, B. Vinter, and M. Leroux, Phys. Rev. B 77, 075308 (2008)

${ }^{26}$ N. Okada, A. Ishikawa, K. Yamane, K. Tadatomo, U. Jahn, and H. T. Grahn, Phys. Status Solidi A 211, 736 (2014).

${ }^{27}$ N. Kriouche n, P. Vennégues, M. Nemoz, G. Nataf, and P. De Mierry, J. Cryst. Growth 312, 2625 (2010).

${ }^{28}$ T. Gühne, Z. Bougrioua, P. Vennéguès, M. Leroux, and M. Albrecht, J. Appl. Phys. 101, 113101 (2007).

${ }^{29}$ P. Vennéguès, F. Tendille, and P. De Mierry, J. Phys. D Appl. Phys. 48, 325103 (2015). 